\title{
ACCURACY ESTIMATION OF A NEW OMNIDIRECTIONAL 3D VISION SENSOR
}

\author{
R. Orghidan, J.Salvi \\ Institute of Informatics and Applications, \\ Computer Vision and Robotics Group, \\ University of Girona, Girona, \\ Edifici P-IV, Av. Lluís Santaló, s/n, \\ 17071 Girona, Catalonia, Spain.$$
\text { \{radu,qsalvi\}@eia.udg.es }
$$

\author{
E.M. Mouaddib \\ Centre de Robotique, Électrotechnique \\ et Automatique, \\ Université de Picardie Jules Verne, \\ 7, Rue du Moulin Neuf \\ 80000 Amiens, France \\ mouaddib@u-picardie.fr
}

\begin{abstract}
We present a computer vision system that associates omnidirectional vision with structured light with the aim of obtaining depth information for a 360 degrees field of view.The approach proposed in this article combines an omnidirectional camera with a panoramic laser projector. The article shows how the sensor is modelled and its accuracy is proved by means of experimental results. The proposed sensor provides useful information for robot navigation applications, pipe inspection, 3D scene modelling etc.
\end{abstract}

\section{INTRODUCTION}

Capturing images of the surrounding scene is an attractive feature for many applications, going from robot navigation to surveillance and virtual tourism, which made omnidirectional vision one of the most active fields of computer vision. Yagi [10] surveyed the existing techniques for building cameras with a wide field of view and Svoboda [9] proposed several classifications of the existing omnidirectional cameras according to their most important features.

The catadioptric sensors enhance the vision field by using reflecting surfaces (convex or planar mirrors) coupled to a conventional camera. These sensors can be classified depending on the way they gather the light rays. The Single View Point (SVP) sensors form the image using only the rays that cross into a point, called focus, as explained by Baker and Nayar in [1]. The catadioptric sensors that do not possess a single focal point (non-SVP) are less used but proved to be helpful for applications with specific requirements as shown by Hicks [3].

Stereo catadioptric sensors are special structures of mirrors and lenses designed for obtaining depth from images

This work is partially supported by the Spanish project CICYT TIC 2003-08106-C02-02 and by the AIRE mobility grant provided by the Generalitat of Catalunya that allowed a four month stay at the CREA lab in Amiens, France with a wide field of view. The results obtained by stereoscopic vision depend on the accuracy of matching the points between the observed images. Structured light based techniques are a particular case of stereo vision where one of the cameras is replaced by a pattern projector [4]. Using this technique is similar to placing visible landmarks in the scene so that the image points can be identified and matched faster and with high accuracy.

This paper presents an omnidirectional sensor that provides 3D information using structured light. The sensor is formed by two components: a catadioptric camera coupled with an omnidirectional structured light projector. By mounting the omnidirectional sensor on a mobile robot applications such as 3D map building or robot navigation and localization can be performed within a horizontal field of view of 360 degrees. The sensor and the models of its two parts are presented in section 2. The components used for building the sensor are described in section 3. The experimental results are shown in section 4 . The article ends with conclusions.

\section{SENSOR MODELLING}

The proposed sensor perceives depth using structured light. Besides, this technique avoids the correspondence problem as explained in the introductory section. The sensor, presented in Figure 1, is formed by an omnidirectional camera coupled to a structured light projector that has a field of view of 360 degrees. The projector sends a circular pattern which is reflected by the conical mirror and becomes a light-stripe on the scene. The parabolic mirror reflects the scene into the camera and the laser-stripe can be immediately identified [5]. With the models for both components of the sensor a precise triangulation can be carried out. 


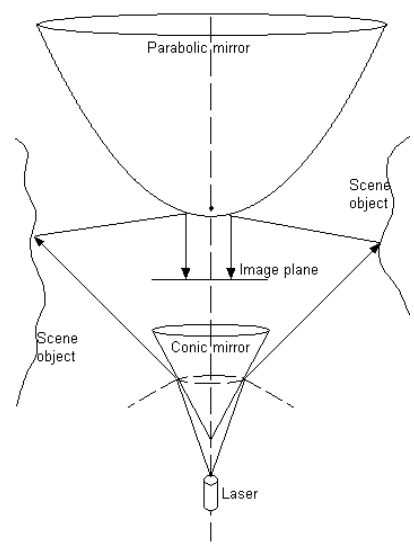

(a)

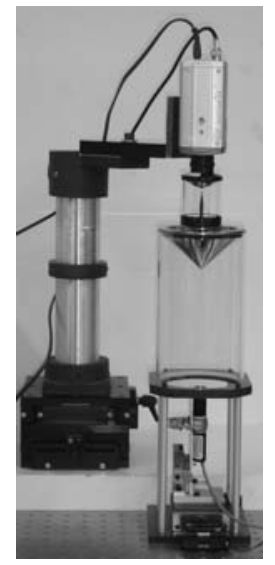

(b)
Fig. 1. The proposed sensor. (a) The model of a catadioptric omnidirectional camera with structured light projector. (b) The laboratory prototype.

\subsection{Omnidirectional camera model}

Assuming that the pair camera-mirror possesses a SVP, the omnidirectional camera can be modelled as the projection onto the sphere followed by the projection to a plane, as we explained in [6]. The calibration is performed using a set of known 3D points, $P_{w}=\left[x_{w}, y_{w}, z_{w}\right]$, distributed on the four walls of a cube placed around the sensor. If $P_{s}=$ $\left[x_{s}, y_{s}, z_{s}\right]$ is the intersection of the light ray emitted by the point $P_{w}$ with the sphere of radius $R$, then the perspective projection of $P_{s}$ on the image plane from a point $C=[0, \xi]$ produces a point $P_{i}=[x, y]$ as expressed in equation (1).

$$
\left\{\begin{array}{l}
u=\frac{\alpha_{u}(\xi+\varphi) x_{w}}{\xi \sqrt{x_{w}^{2}+y_{w}^{2}+z_{w}^{2}}-z_{w}}+u_{0} \\
v=\frac{\alpha_{v}(\xi+\varphi) y_{w}}{\xi \sqrt{x_{w}^{2}+y_{w}^{2}+z_{w}^{2}}-z_{w}}+v_{0}
\end{array}\right.
$$

The parameters of the model are $\xi$, which depends on the eccentricity; $\varphi$ which is a function of both the eccentricity and the scale; $\alpha_{u}, \alpha_{v}, u_{0}, v_{0}$, the intrinsic camera parameters; $r_{X}(\phi), r_{Y}(\theta), r_{Z}(\varphi)$, and $t_{x}, t_{y}, t_{z}$, the six extrinsic parameters that model respectively the orientation and the translation between the world coordinate system placed in the upper corner of the first calibration plane and the camera coordinate system. The orientation vectors are functions of the three angles $(\phi, \theta, \varphi)$ which define the rotation on each axis and are expressed in radians while the translations are measured in millimeters, as detailed in [8].

\subsection{Omnidirectional laser projector model}

The omnidirectional light projector is formed by a laser which emits a circular pattern and is pointed to a conical mirror so that the projected light covers the entire field of view of the catadioptric camera, as shown in Figure 2.a. The 3D coordinates of the points of the light stripe projected on the known planes placed around the sensor can be determined and used for calibrating the pair laser-mirror.

The quadratic surface that fits all bright spots with known coordinates $P_{w i}(x, y, z)$ is represented in eq. 2 . A method for finding its parameters is shown in [6].

$$
\left[\begin{array}{l}
x \\
y \\
z
\end{array}\right]^{\prime} \cdot\left[\begin{array}{lll}
a_{11} & a_{12} & a_{13} \\
a_{21} & a_{22} & a_{23} \\
a_{31} & a_{32} & a_{33}
\end{array}\right] \cdot\left[\begin{array}{l}
x \\
y \\
z
\end{array}\right]+\left[\begin{array}{l}
x \\
y \\
z
\end{array}\right]^{\prime} \cdot\left[\begin{array}{l}
\beta_{1} \\
\beta_{2} \\
\beta_{3}
\end{array}\right]+f=0
$$

\section{SENSOR DESCRIPTION}

Due to their optical properties, catadioptric sensors having a SVP were chosen for building the omnidirectional camera of the proposed sensor. Commonly, the central projection constraint is fulfilled by the use of a parabolic mirror in conjunction with an orthographic camera or a hyperbolic mirror with a perspective camera. The first configuration that preserves the SVP independently of the translation between the mirror and the camera, was finally preferred.

The mirror used for the laser projector has a conical shape. On vertical section, the conical mirror behaves like a planar mirror and consequently provides a much better resolution than any other omni-mirror shape. Moreover, the curved mirrors (such as parabolic, hyperbolic, etc.) increase defocus blur because of their bend. Consequently, the cone bears out to be the ideal shape of mirror to be used for building the structured light projector.

The system was build using off the shelf components. The optics and the mirror used for the omnidirectional camera were provided by Remote Reality [7]. The camera is a Sony SSC-DC198P with the ccd of 1/3". The laser and its optics are produced by Lasiris, the diode power is $3 \mathrm{~mW}$ and produces red light with a wavelength of $635 \mathrm{~nm}$.

\section{EXPERIMENTAL RESULTS}

The experiments presented in this section are aimed to findout the reliability of the sensor for robot navigation tasks by evaluating its accuracy of measuring depth in a real scene. The section starts with an overview of the sensor calibration. Depth measurements are performed using a laboratory setup and the results are presented and commented in the second part of the current section.

\subsection{Sensor calibration}

The camera calibration is performed using a set of 285 dots distributed on the four planes placed around the sensor. The 
distance between the centers of any two adjacent dots on the same plane is $6 \mathrm{~cm}$ and the height of the calibration plane is $80 \mathrm{~cm}$. A semi-automatic point extraction method is performed. The centers of several dots, selected manually by the user, are determined with sub-pixel accuracy. The centers of the other dots on the same plane are automatically found, with the same precision. The calibrated parameters of the camera-model are listed in the Table 1 . The average calibration error is $\mu=2.2 \mathrm{px}$ and the sample standard deviation $\sigma=2.542$.

The conical mirror used for building the laboratory prototype has a height $h=4.4 \mathrm{~cm}$ and the cone aperture angle is $\beta=52$ degrees. The laser projects a circular cone with a fan angle $\alpha=11.4$ degrees. Since the relation between the two angles is $\beta \approx 0.5(\alpha+\pi / 2)$ the laser is reflected along a very flat surface which can be approximated to a plane: $a x+b y+c z+d=0$, see Figure 2.b. In the omnidirectional image, shown in Figure 2.a, the center of the laser stripe is determined with sub-pixel accuracy using the peak detection method described by Forest [2]. The intersections between the laser plane and the planes of the calibration box are straight lines which are imaged as elliptical arcs by the omnidirectional camera. Using this information the calibration accuracy is increased by performing an ellipse fitting for each line in the scene, as shown in Figure 3. The 3D

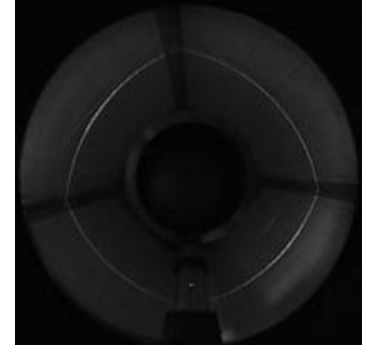

(a)

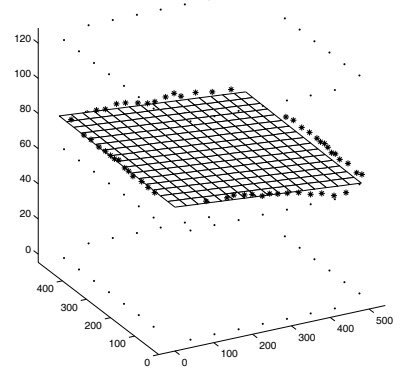

(b)
Fig. 2. Omnidirectional laser projector calibration. (a) Omnidirectional view of the laser pattern. (b) Plane fitted to a set of points of the laser stripe. The dotted rectangles are the points on the planes around the camera.

positions of the laser points are calculated using the reversed camera model for the back-projection of the image points on the known calibration planes. Finally, the four parameters of the laser plane are calculated: $a=0.0006, b=0.0053$, $c=1$ et $d=82.2661$.

\subsection{Depth perception}

Depth is calculated by triangulation. The laser projection is detected in the omnidirectional image with sub-pixel accuracy. Since the observed light spots belong to both the laser

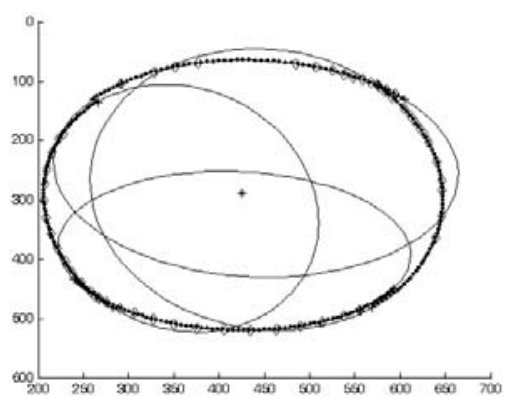

Fig. 3. Ellipse fitting to laser points in the omnidirectional image.

pattern and to the rays crossing in the mirror focal point their 3D position can be calculated. In order to have an estimation of the sensors' accuracy we placed a planar surface at different known positions and its location was estimated using the proposed sensor. The points of the laser stripe, imaged by the omnidirectional camera, were mapped to 3D points. The 10 positions of the plane used in the experimental setup, the cloud of points obtained given by the sensor for each position of the plane and the camera coordinate system are shown in Figure 4.

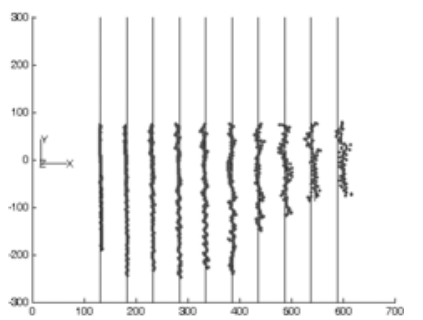

(a)

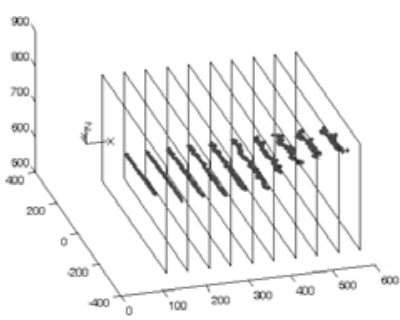

(b)
Fig. 4. Depth perception for 10 positions of a plane. (a) Upper view. (b) Lateral view.

The mean error is the average of the distances of the 3D points to the reference plane. The error was calculated for 10 positions of the plane which was placed at known distances from the sensor within a range going from $132 \mathrm{~mm}$ to $590 \mathrm{~mm}$. The evolution of the mean error and the standard deviation for each position of the plane are presented in Figure 5. The mesurable quantities represented on both axes are expressed in millimeters.

Analyzing the results it can be seen that the depth perception accuracy is high in the proximity of the sensor: the points of the planar surface are detected with an average error of $1 \mathrm{~mm}$ at a distances up to $28 \mathrm{~cm}$ from the sensor. The accuracy decreases with the distance but the information is still reliable even for an object placed at more than $50 \mathrm{~cm}$ away from the sensor since its shape is detected with an 
Table 1. The calibrated parameters for the omnidirectional camera. (The measure units are: the intrinsic parameters, in pixels; the angles, in degrees; the translations and the mirror parameters, in mm). Average error: 2.2 pixels.

\begin{tabular}{|cccccccccccc|}
\hline$\xi$ & $\varphi$ & $\alpha_{u}$ & $\alpha_{v}$ & $u_{0}$ & $v_{0}$ & $r_{x}$ & $r_{y}$ & $r_{z}$ & $t_{x}$ & $t_{y}$ & $t_{z}$ \\
\hline 1.06 & -9.64 & -32.53 & 33.24 & 429.51 & 292.72 & 0.02 & 0.01 & -0.009 & -26.45 & -0.82 & -754.1 \\
\hline
\end{tabular}

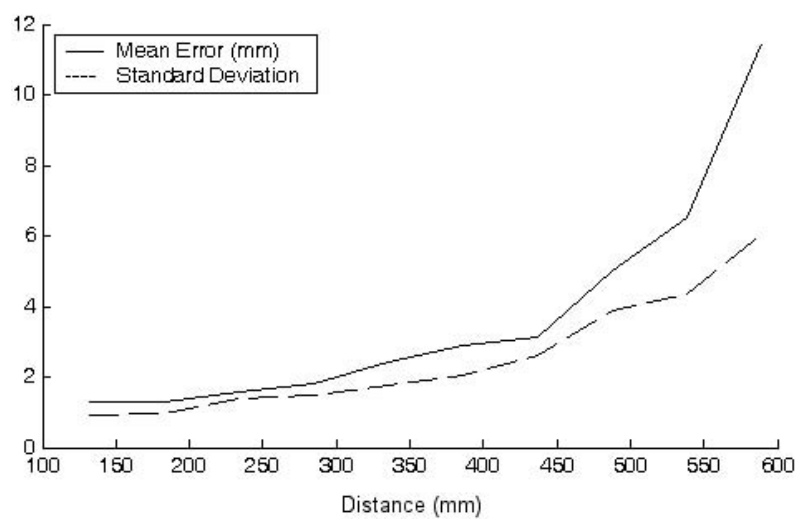

Fig. 5. Accuracy of the proposed sensor. The solid line follows the error distance from the mapped 3D points to the reference plane. The dotted line represents the standard deviation at each position of the plane.

average accuracy of $6 \mathrm{~mm}$. In all the cases, the maximum error obtained represents less than $2 \%$ of the distance from the sensor to the planar surface which proves that the sensor can be successfully used in robot navigation tasks. Moreover, when previous information about the scene geometry is available the accuracy can be increased by using this information at the segmentation stage for removing potential noise sources.

\section{CONCLUSIONS}

This article describes the prototype of a computer vision sensor capable to estimate depth. The two omnidirectional systems that compose the camera are calibrated and the resulting model is used for measuring distances in a real scene. The experimental results show that the accuracy of the sensor is less than $2 \%$ for obstacles placed in a range of $59 \mathrm{~cm}$ in any direction. The results obtained are encouraging and prove that this sensor can be used in real robot navigation and depth perception applications. The use of 360 degrees images and scene-depth information is ideal for robot navigation tasks and especially in obstacle avoidance and map building.

\section{REFERENCES}

[1] S. Baker and S.K. Nayar. A theory of catadioptric image formation. IEEE Int. Conf. on Computer Vision, ICCV, pages 35-42, 1998.

[2] J. Forest, J. Salvi, E. Cabruja, and C. Pous. Laser stripe peak detector for $3 \mathrm{~d}$ scanners. a fir filter approach. In International Conference on Pattern Recognition, volume 3, pages 646 - 649, Cambridge, United Kingdom, August 2004.

[3] R.A. Hicks and R.K. Perline. Geometric distributions for catadioptric sensor design. In CVPRO1, pages I:584-589, 2001.

[4] J. Batlle. J. Salvi, J. Pagés. Pattern codification strategies in structured light systems. Pattern Recognition 37, 4:827-849, April 2004.

[5] Radu Orghidan, Joaquim Salvi, and El Mustapha Mouaddib. Calibration of a structured light-based stereo catadioptric sensor. Workshop on Omnidirectional Vision, IEEE Conf. on Computer Vision and Pattern Recognition - Volume 7, 2003.

[6] Radu Orghidan, Joaquim Salvi, and El Mustapha Mouaddib. Omnidirectional depth computation from a single image. IEEE International Conference on Robotics and Automation, ICRA, April 18-22, 2005.

[7] Remote Reality. http://www.remotereality.com/.

[8] J. Salvi, X. Armangue, and J. Batlle. A comparative review of camera calibrating methods with accuracy evaluation. Pattern Recognition, 35(7):1617-1635, July 2002.

[9] Tomas Pajdla Tomas Svoboda. Panoramic cameras for $3 \mathrm{~d}$ computation. Proceedings of the Czeck Pattern Recognition Workshop, pages 63-70, February 2000.

[10] Y. Yagi. Omnidirectional sensing and its aplications. IEICE Trans on Information and Systems, E82D(3):568-578, 1999. 